In Situ

Revue des patrimoines
In Situ

Revue des patrimoines

17 | 2011

Les patrimoines de l'enseignement supérieur

\title{
Universeum
}

\section{Sébastien Soubiran}

\section{OpenEdition}

\section{Journals}

Édition électronique

URL : http://journals.openedition.org/insitu/10205

DOI : 10.4000/insitu.10205

ISSN : 1630-7305

\section{Éditeur}

Ministère de la culture

\section{Référence électronique}

Sébastien Soubiran, «Universeum », In Situ [En ligne], 17 | 2011, mis en ligne le 10 avril 2013, consulté le 20 avril 2019. URL : http://journals.openedition.org/insitu/10205 ; DOI : 10.4000/insitu.10205

Ce document a été généré automatiquement le 20 avril 2019.

\section{(2) $\odot \Theta \Theta$}

In Situ Revues des patrimoines est mis à disposition selon les termes de la licence Creative Commons Attribution - Pas d'Utilisation Commerciale - Pas de Modification 4.0 International. 


\title{
Universeum
}

\author{
Sébastien Soubiran
}

UNIVERSEUM ${ }^{1}$, « réseau européen pour le patrimoine universitaire » est aujourd'hui une association à but non lucratif régie par le Code civil local maintenu en vigueur dans les départements du Bas-Rhin, Haut-Rhin et Moselle ${ }^{2}$. Ce réseau européen à l'origine principalement dédié aux musées et collections universitaires a été créé à l'initiative de l'université de Halle-Witternberg alors engagée dans la préparation d'une importante exposition destinée à marquer les 500 ans d'existence de l'Université de Wittenberg (2002). En engageant le dialogue avec d'autres universités européennes, cette préparation a permis de mettre en avant l'existence d'une grande quantité de collections universitaires, relativement dispersées les unes par rapport aux autres et en état de conservation et d'inventaire très divers. Il apparaissait également que les musées universitaires européens étaient confrontés à de graves problèmes structuraux souvent identiques. La liste de ces problèmes est malheureusement bien connue : c'est d'abord l'accès difficile pour un large public intéressé, c'est également les conditions de conservation souvent précaires des spécimens, ou encore l'absence d'une documentation numérisée et d'informations électroniques sur les collections ou leur inventaire, mais c'est aussi la disparition de postes pour les personnes en charge de la préservation de ces collections ou carrément des structures muséales elles-mêmes dans le cadre des restructurations politiques des universités alors en cours en Europe. 
Figure 1

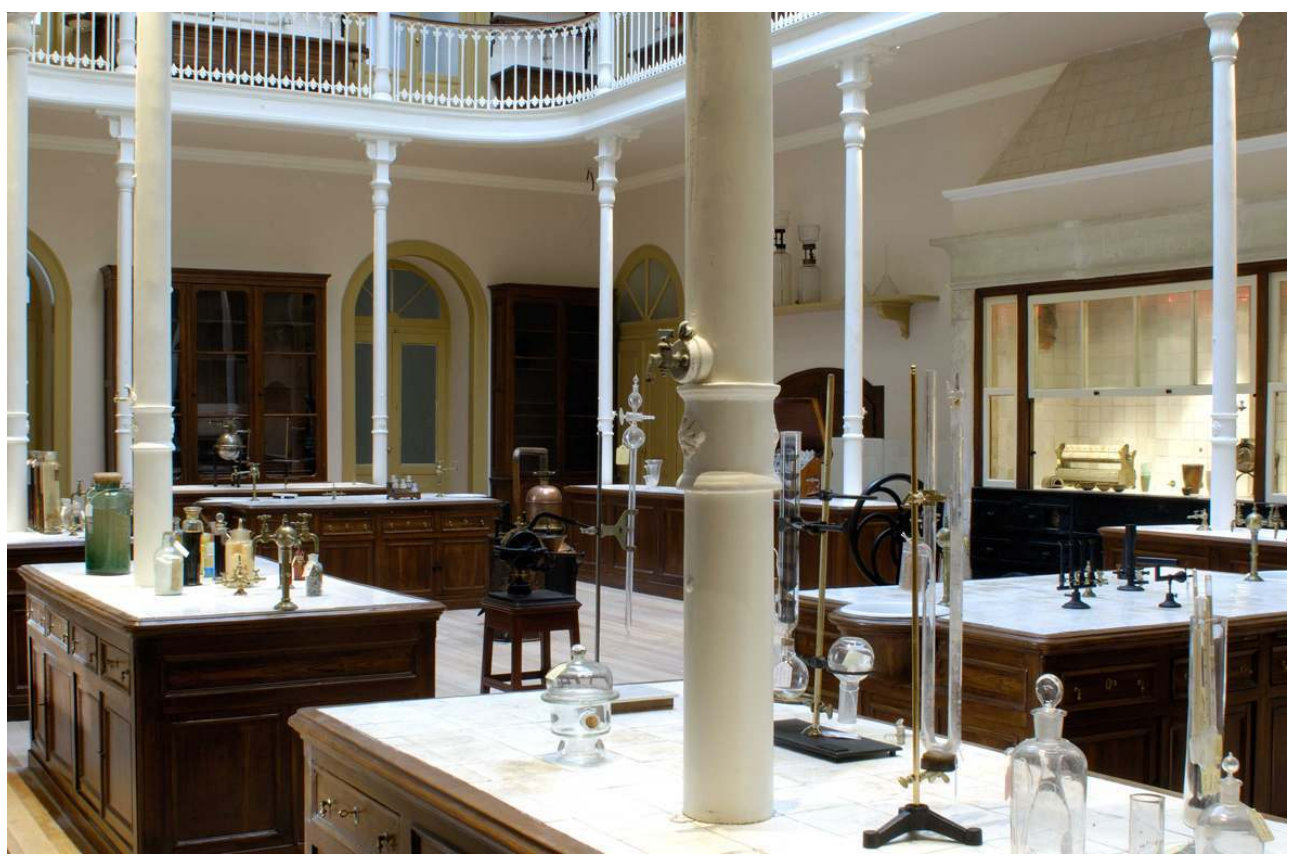

Laboratoire de Chimie de l'Ecole polytechnique construit en1890, Musée de Science de l'Université de Lisbonne.

Phot. P. Cintra, 2007, avec l'autorisation du Musée de Science de l'Université de Lisbonne.

UNIVERSEUM est alors créé lors de l'organisation d'une conférence organisée à Halle en avril 2000, à laquelle participent les responsables de musées et collections de 12 des universités européennes les plus anciennes (Amsterdam, Berlin, Bologne, Cambridge, Groningen, Halle, Leipzig, Londres, Oxford, Pavie, Uppsala, Utrecht). La première action d' UNIVERSEUM est politique, avec la rédaction d'un texte commun formulant des exigences essentielles pour la mise en œuvre d'une politique globale (des universités, des gouvernements nationaux et des instances européennes) afin d'améliorer la conservation et l'accès au public du patrimoine académique de chacune des universités présentes. L'impact de la "déclaration de Halle" sur les instances européennes alors en pleine réflexion sur la question du patrimoine des universités en Europe ${ }^{3}$ se matérialise par la publication d'une recommandation du Conseil de l'Europe en $2002^{4}$. 


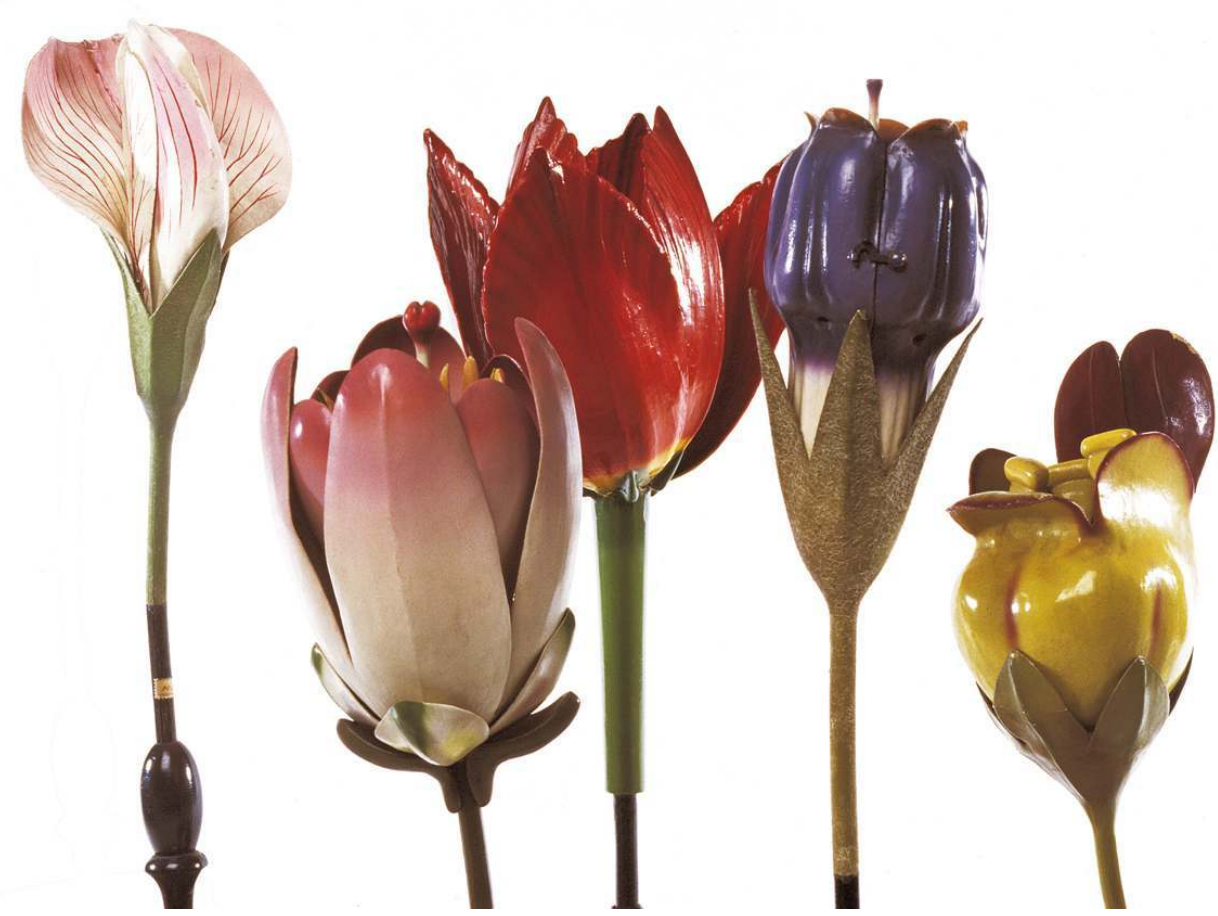

Modèles agrandis de fleurs, matériel pédagogique démontable en papier mâché peint à l'huile, fabriqués par Robert et Reinhold Brendel à Breslau, puis Berlin de 1866 à 1925. Université de Strasbourg.

(C) Bernard Braesch, UDS.

Au-delà de l'Europe, les préoccupations exprimées par univERSEum trouvent un écho au sein de la communauté internationale des musées, avec la création au cours de la $19^{\mathrm{e}}$ assemblée générale de l'ICOM, à Barcelone en juillet 2001, d'un nouveau comité thématique international dédié aux musées et collections universitaires (UMAC). La création d'UMAC témoigne de la reconnaissance de l'identité spécifique des musées universitaires par la plus importante organisation internationale de ce domaine ${ }^{5}$. Après avoir fonctionné comme un réseau informel pendant 10 ans, UNIVERSEUM s'est structuré en association, lors de sa conférence annuelle organisée à Uppsala en 2010, afin de renforcer et approfondir sa mission. Les nouveaux statuts et objectifs résultent des discussions poursuivies pendant deux ans, au cours des réunions annuelles et dans le cadre d'un groupe de travail réunissant sept responsables de collections et musées universitaires de cinq pays européens. Ils se veulent résolument complémentaires de ceux d'UMAC, d'une part en concentrant les actions au niveau de l'Europe et d'autre part en s'intéressant à la préservation, l'étude, l'accessibilité et la valorisation du patrimoine universitaire européen compris au sens large, matériel et immatériel (collections, musées, archives, bibliothèques, jardins botaniques, observatoires astronomiques, monuments, etc.). 




Prothèse en bois fabriquée par le chirurgien Ferdinand Sauerbruch (1875-1951), Musée d'histoire médicale de Berlin, Hôpital universitaire de la Charité.

(c) Université Humboldt, Berlin.

UNIVERSEUM compte aujourd'hui plus de 60 membres individuels et institutionnels de 17 pays européens ${ }^{6}$. Le nombre croissant de participants aux conférences annuelles et la richesse des présentations proposées témoignent de la vitalité des activités de recherche et de valorisation sur le patrimoine des universités. Cette dynamique est nécessaire pour renforcer sa visibilité et soutenir une action de lobbying auprès des instances politiques nationales et européennes. Les recommandations publiées par la Wissenschaftsrat, conseil national scientifique allemand, en janvier 2011, démontrent que la mise en place d'un réseau porté par des projets structurants peut conduire à une reconnaissance par des instances académiques ${ }^{7}$. C'est sur la base des actions menées par les collègues allemands - et en particulier Cornelia Weber de l'Université Humboldt à Berlin (bases de données, projets de recherches, conférences), que le Conseil scientifique national allemand a publié une liste de recommandations par laquelle non seulement il encourage les universités à préserver leurs collections scientifiques mais souligne également le fort potentiel de recherches de ces collections. Fort de cet exemple, UNIVERSEUM entend participer à étendre cette reconnaissance dans l'ensemble des pays européens et obtenir de la Commission européenne des financements pour structurer une véritable politique de recherche, de valorisation et de préservation du patrimoine des universités. 


\section{NOTES}

1. - Article rédigé sur la base des documents de travail et articles écrits avec les membres par intérim du board d'Universeum: Thomas Bremer, Université de Halle-Wittemberg, Président, Marta Lourenco, Université de Lisbonne et Sofia Talas, Université de Padoue, Vice-présidentes, Laeticia Maison, Université de Bordeaux 3, trésorière, Klaus Staubermann, Université d'Edimburg et Roland Wittje, Université de Regensburg, membres ex-officio. Voir notamment, SOUBIRAN, S., LOURENCO, M., WITTJE, R., TALAS, S. \& BREMER, T. « Universeum un réseau pour le patrimoine universitaire en Europe ». La lettre de l'OCIM, 2009, n¹23, p. 5-14.

2. - Un ensemble d'informations concernant Universeum est disponible sur le site: http:// www.universeum.it/.

3. - SANZ, N. \& BERGAN, S. (ed.). Le patrimoine des universités européennes. Strasbourg : Conseil de l'Europe, 2002.

4. - https://wcd.coe.int/wcd/ViewDoc.jsp?Ref=Rec\%282005\%2913\&Language=lanFrench.

5. - Pour plus d'informations voir le site : http://publicus.culture.hu-berlin.de/umac/.

6. - Pour devenir membre d'Universeum télécharger le formulaire sur le site http:// www.universeum.it/ et l'envoyer à s.soubiran@unistra.fr.

7. - Le texte en allemand est disponible à l'adresse : http://www.wissenschaftsrat.de/download/ archiv/10464-11.pdf; un article en anglais dans Nature reprend les points importants : http:// www.nature.com/nature/journal/v470/n7332/full/470005b.html.

\section{AUTEUR}

\section{SÉBASTIEN SOUBIRAN}

Jardin des Sciences, Université de Strasbourg, secrétaire d'Universeum s.soubiran@unistra.fr 